\title{
PERFORMANCE ASSESSMENT OF ANFIS APPLIED TO FAULT DIAGNOSIS OF POWER TRANSFORMER
}

\author{
Nandkumar Wagh ${ }^{1}$ and D.M. Deshpande ${ }^{2}$ \\ ${ }^{1}$ Associate Professor, Electrical Engineering Department, DES's College of Engineering \\ and Technology, Dhamangaon (India) $-444709^{2}$ Former Professor, Department of \\ Electrical Engineering, M. A. N. I .T., Bhopal (India)-462003
}

\begin{abstract}
Continuous monitoring of Power transformer is very much essential during its operation. Incipient faults inside the tank and winding insulation needs careful attention. Traditional ratio methods and Duval triangle can be employed to diagnose the incipient faults. Many times correct diagnosis due to the borderline problems and the existence of multiple faults may not be possible. Artificial intelligence (AI) techniques could be the best solution to handle the non linearity and complexity in the input data. In the proposed work, adaptive neuro fuzzy inference system (ANFIS), is utilized to deal with 9 incipient fault conditions including healthy condition of power transformer with sufficient DGA transformer oil samples. Comparison of the diagnosis performance of both the methods of ANFIS and the feasibility pertaining to the problem is presented. Diagnosis error in classifying the oil samples and the network structure are the main considerations of the present study.
\end{abstract}

\section{KEYWORDS:}

Fault Diagnosis, Power Transformer, ANFIS, GENFIS, Membership Functions

\section{INTRODUCTION}

The reliability of the transmission and distribution network depends upon the healthy condition of Power transformer. It is to be continuously monitored and maintained during its operating conditions. Thermal and electrical stresses mainly cause the incipient faults which further leads to failure of the equipment. Dissolved gas analysis (DGA) has been preferred as the best preliminary diagnosis tool. For diagnosis using DGA, ratio methods such as Rogers ratio, Doernenburg ratio, IEC ratio and Duval triangle has been established as some of the standards. In the situation of multiple faults, the ratio methods fail to diagnose the faults. Among the existing methods, DGA is the most popular and successful method for identifying the incipient faults [1]-[3].Due to the presence of an incipient fault such as overheating or discharge inside the transformer, a corresponding characteristic amount of gases are evolved and gets dissolved in the transformer 
oil. DGA method may be used to determine the type of the fault in a transformer by analysing the concentrations of dissolved gases, rate of gas generation and the ratio of certain gases,. The hydrocarbon gases dissolved in transformer oil are $\mathrm{H}_{2}, \mathrm{CH}_{4}, \mathrm{C}_{2} \mathrm{H}_{2}, \mathrm{C}_{2} \mathrm{H}_{4}, \mathrm{C}_{2} \mathrm{H}_{6}, \mathrm{CO}_{2}$ and $\mathrm{CO}$.

The key gas method [2] is based on determination of the key gas evolved during the fault. The particular gas evolved is related to certain temperature and the fault. During the severity of a fault, all of the gas concentrations will be high, and is not possible to determine the fault using IEEE standard .The artificial intelligence if incorporated taking the benefit of preliminary DGA methods can deliver most accurate results. An integrated approach of neural fuzzy in which subtractive clustering is mainly used to deal with the transformer fault diagnosis. Good diagnosis accuracy is registered using the approach and the performance comparison with the conventional methods is also presented [3]. Neural network mapping into a rule-based fuzzy inference system is proposed by transforming it into a set of rules using IEC 60599. This leads to knowledge extraction which is further used to generate new rule table for better fault diagnosis so as to cover the input space beyond ratio suggested in the literature of DGA [4].

The neural network has good classification ability and handling the noisy data. The artificial intelligence techniques, such as neural networks and fuzzy set theory have been applied by many researchers to enhance the diagnosis accuracy [5]. The diagnosis results of fuzzy logic using different standards such as IEC, CEGB and ASTM are presented with limited samples of transformers [6]. The diagnosis of transformer faults using ANFIS with 10 fuzzy rules and IEC ratio is proposed [7].

The fuzzy systems, can handle the problems involving uncertainties, but has no learning ability of previous diagnosis results. It is not possible further to adjust the diagnostic rules automatically. Fuzzy systems and neural networks are the two different information processing systems. Knowledge representation can be done very well using fuzzy system, while neural networks are capable of learning from experiences. A novel transformer fault diagnosis using fuzzy C-Means clustering and RBF neural network in which fuzzy clustering is proposed in [8] to select the efficient training data and reduction in learning process time. Mainly the degree and origin of ageing using RBF network is investigated under normal and care condition of the transformer.

Three new learning algorithms for modeling the TSK fuzzy system based on training error and genetic algorithm are utilized [9]. In the first phase initial structure of neural fuzzy network is created by optimum point estimation from the input output space using KNN (K-Nearest Neighbor) and Mean shift method for the second algorithm keeping adding the new neurons by error based algorithm. Then redundant neurons are recognized and removed using GA and the third algorithm builds the network in one phase using modified version as per the earlier algorithms. No rule reduction is made in the method. However the fuzzy sets have been optimized with as many data samples in the input. One of the problems associated with the fuzzy expert system is the human expertise in constructing the rules.

A method for simultaneous diagnosis of fault type and fault location which uses an adaptive neuro- fuzzy inference system based on DGA is proposed [10]. Using IEC 599 standards, ANFIS has been trained on the data of 6 transformers initially. To locate the fault position and its type an additional input data of $\mathrm{CO} 2 / \mathrm{CO}$ ratios has been used. Comparison of the system performance is presented using ANN and some other methods. 
Unsatisfactory performance of Rogers, Doernenburg, and NBR7274 methods has been registered, though these methods are widely accepted by the utilities [11].The results provided by the neural network applied alone are limited. Its performance, however, could be improved with the use of a larger number of representative examples in its training phase. Fuzzy system approach has proven better for the problem proposed by the researcher.

The problem of rule determination by human expertise is taken care by TSK model. This model automatically generates the rules based on the membership functions and the input parameters with target are proposed for enhancing the performance of the diagnostic system. Reduction in the number of rules and simplicity with better diagnosis results are the main objectives of this work. Nine incipient fault conditions including a healthy condition of transformer oil insulation are considered in this work and the DGA data of oil insulation of power transformer is utilized. The work is especially focussing on the incipient fault detection with better accuracy so that no sample in the database remains undetected.

\section{ADAPTIVE NEURO-FUZZY INFERENCE SYSTEM (ANFIS)}

\subsection{Membership Functions (MF's)}

The MF's of input plays an important role in deciding the number of rules and the further output in diagnosis. Various types of MF's are available in fuzzy tool box of matlab. Fuzzy logic deals with fuzzy sets defined in the universe of discourse ' $U$ '. Fuzzy set can be determined by a membership function by mapping the members of ' $U$ ' to a membership range from $0-1$ and translate uncertain information into specific and related data [12]. The important step in applying fuzzy logic and inference is the selection of membership functions (MF's) for the input. Triangular, trapezoidal, bell, g-bell, Gaussian, Gaussian 2 etc. are the membership functions available in mat lab. The parameters of the MF's play an important role in inference process. These parameters can be initially selected and modified in the training process. Sometimes optimization of its parameters leads to better solution of the problem.

A 3 parameterized, generalized bell (G Bell) MF specified can be expressed as:

$$
\text { bell }(x ; a, b, c)=1 /(1+|x-c / a| 2 b) \text {, }
$$

It is a direct generalization of the Cauchy distribution used in probability theory. To vary the center and width of MF $\mathrm{c}$ and a can be adjusted, but to control the slopes at the crossover points, $\mathrm{b}$ has to be adjusted. It is shown in fig.1.

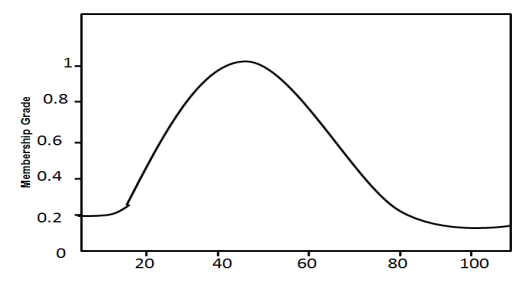

Fig. 1. G-Bell MF 
Although the Gaussian and bell MF's achieve smoothness, they are unable to specify the asymmetric MF's important in certain applications. Other MF's can also be specified with its parameters and shape.

\subsection{Structure of System and Rules}

The best features of fuzzy systems and neural Networks are combined in ANFIS. In this approach the network topology is used to reduce the optimization search space performed by fuzzy system. Tuning of the fuzzy controller parameters is performed by neural network. The actual process of mapping from a given input to an output using fuzzy logic is performed in fuzzy inference. This process involves membership functions for input and output, fuzzy logic operators and if-then rules. Fig. 2 shows the architecture of fuzzy inference system (FIS).

The most important methods used in the FIS are Mamdani and Takagi-Sugeno-Kang method (TSK). Consequent of fuzzy rules is the main difference among the two methods. In the proposed work, fuzzy rules are generated from the input output dataset of 563 DGA samples of power transformer using TSK method of FIS available in fuzzy tool box of matlab.

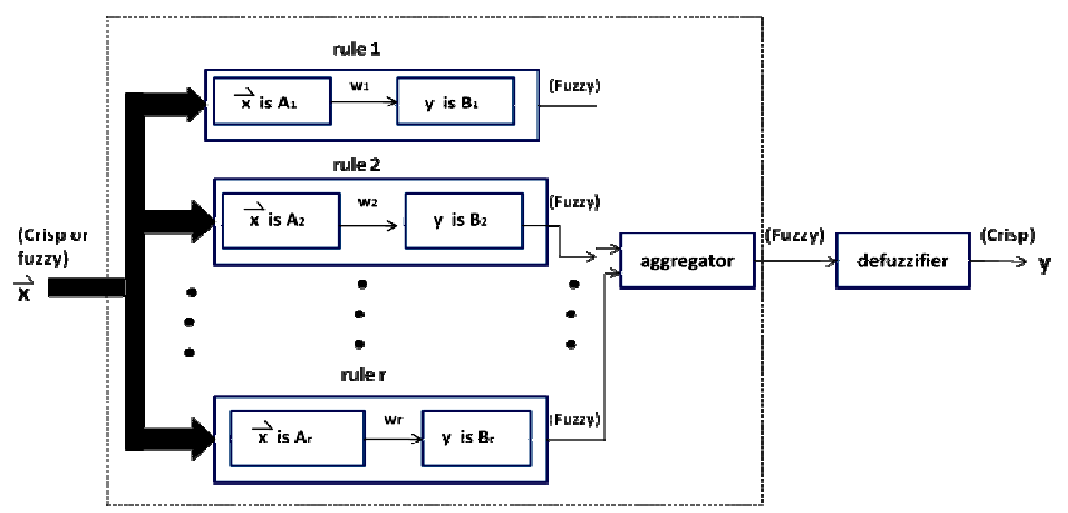

Fig. 2. Architecture of FIS

This model [13] combines fuzzy sets in antecedents and the crisp function in output as shown below,

IF ( $\mathrm{x} 1$ is $\mathrm{A})$ AND ( $\mathrm{x} 2$ is $\mathrm{B})$ THEN $\mathrm{y}=\mathrm{f}(\mathrm{x} 1, \mathrm{x} 2)$

IF $\mathrm{X}$ is small THEN $\mathrm{Y} 1=4$

IF $\mathrm{X}$ is medium THEN $\mathrm{Y} 2=-0.5 \mathrm{X}+4$

IF $\mathrm{X}$ is large THEN $\mathrm{Y} 3=\mathrm{X}-1$

A complicated hidden relationship between the concentration of gases and the fault can be handled using fuzzy set theory. In the proposed methodology, low $(\mathrm{L})$, medium $(\mathrm{M})$, high $(\mathrm{H})$ linguistic variables are used to represent the concentration of gases .Automatically the rules of relationship are generated using 5 types of MF's for input gases concentrations and a single output and the inference regarding the particular fault is made.

If then rules of the FIS are formulated and are of the form.

If $\mathrm{MH}=\mathrm{M}$ and $\mathrm{AE}=\mathrm{M}$ and $\mathrm{EE}=\mathrm{L}$ and $\mathrm{EM}=\mathrm{H}$, then condition $\mathrm{C}$. Similarly other rules can also be formulated as per the experience and knowledge base.

Fuzzy inferences in the form of rules using max/min composition are, 
Rule $1=\min \{\mathrm{MH}=\mathrm{M}, \mathrm{AE}=\mathrm{M}, \mathrm{EE}=\mathrm{L}, \mathrm{EM}=\mathrm{H}\}$

Rule $2=\min \{\mathrm{MH}=\mathrm{H}, \mathrm{AE}=\mathrm{M}, \mathrm{EE}=\mathrm{L}, \mathrm{EM}=\mathrm{L}\}$

Rule $\mathrm{n}=\min \{\mathrm{MH}=\mathrm{VH}, \mathrm{AE}=\mathrm{L}, \mathrm{EE}=\mathrm{H}, \mathrm{EM}=\mathrm{L}\}$

The consequent part will specify the fault condition,

Condition $\mathrm{C} 1=\max \{$ rule1, rule2 $\}$

Condition $\mathrm{C} 2=\max \{$ rule $\mathrm{r}$, rule $\mathrm{p} . \ldots . .$. rule $\mathrm{n}\}$.

' $N$ ' number of rules can be generated either by experience of the researchers or automatically using the modules of ANFIS.

\subsection{Algorithms}

Sugeno (TSK) and Mamdani are the commonly used fuzzy inference systems. The FIS structure is constructed using these models. To partition the input space and to construct the FIS for a particular application are of great concern. The antecedent and the consequent part of the rules define the fuzzy region and the behavior of various constituents in that region. The consequent constituent can be a consequent MF as defined by Mamdani and a constant value as used in Sugeno model.

Different fuzzy inference systems, having same antecedents but different consequent constituents can also be formulated.

To tackle such problems, the method of input space partitioning to form the antecedents of the fuzzy rules is applicable to all the models of FIS. Hence the methods and the algorithms used to create the FIS structure and rules are necessary.

Clustering algorithms are used extensively to organize and categorizing the data, model construction and data compression. The off line clustering techniques frequently used with fuzzy modeling and RBF networks are elaborated. This technique partitions the data into several groups such that the similarity within a group is larger than that of other groups. Similarity metrics showing the similarity in the groups must be used. Input variables must be normalized to within the unit interval $(0,1)$ as; most similarity metrics are sensitive to the ranges of elements in the input vectors.

The various methods and algorithms used in partitioning and clustering the data [12] are described below.

\subsubsection{Grid Partitioning}

This method is often chosen in designing the fuzzy controller, which usually involve many state variables as input to the controller. This needs less number of MF's for each input. But if the numbers of inputs are more, there is a problem that the rules generated shall be in large number. If $3 \mathrm{MF}$ 's are used in the fuzzy model with 7 inputs, then the numbers of rules generated are $3^{7}=$ 2187. In the proposed work, GENFIS1 which generates the grid structure is used. As many inputs and membership functions may generate more number of rules. This partitioning is shown in figure 3 . 


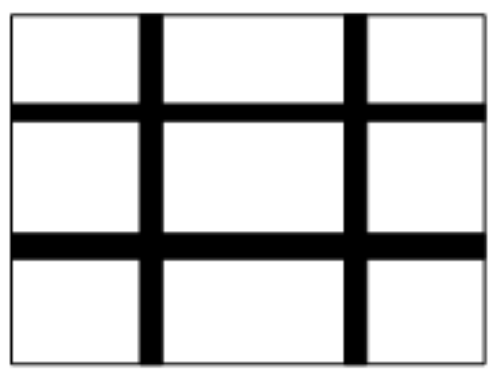

Fig. 3.Grid partitioning

\subsubsection{Subtracting clustering}

This algorithm is proposed by Chiu, in which data points are considered as the candidates for cluster centers. The computation depends upon the number of data points but independent of the dimension of the problem. Consider a collection of $n$ data points $\left\{\mathrm{x}_{1} \ldots \mathrm{x}_{\mathrm{n}}\right\}$ in $M$-dimensional space. The data points are assumed to have been normalized within a hypercube. Since each data point is a candidate for cluster centers, a density measure at that point $\mathrm{x}_{\mathrm{i}}$ is defined as,

$D_{i}=\sum_{j=1}^{n} \exp \left(-\frac{x_{i}-x_{j}^{2}}{\left.\frac{y_{n}}{2}\right)^{2}}\right)$,

Where, ' $r_{2}$ ' is a positive constant. Density value of data point depends upon neighboring data points. The radius ' $r a$ ' defines a neighborhood and the data points outside this radius may not contribute more to the density measure.

The data point with the highest density measure is selected as the first cluster center after the density measure of each data point has been calculated.

If $D_{c 1}$ is density measure for data point $X_{c 1}$, then the density measure for each data point $x_{i}$ can be revised using the relation as shown.

$$
D_{i}=D_{i}-D_{c l} \exp \left(-\frac{x_{i}-x_{2 i}^{2}}{\frac{\gamma_{2} y^{2}}{y^{2}}}\right)
$$

Where, ' $\mathrm{r}_{\mathrm{b}}$ ' is a positive constant.

It is seen that, the data points near the first cluster center $x_{c 1}$ will have significantly reduced measures. The constant ' $r_{b}$ ' is normally larger than ' $r_{a}$ ' to prevent closely spaced cluster centers.

After revision of the density measure for each data point, the next cluster center $\mathrm{x}_{\mathrm{c} 2}$ is selected and all of the density measures for other data points are revised again. Recalculations or revision is made and repeated till sufficient numbers of cluster centers are generated. In subtractive clustering applied to input-output data; each of the cluster centers represents a prototype that exhibits certain characteristics of the system to model. These cluster centers can then be used as the centers for the fuzzy rules' premise in a zero-order Sugeno fuzzy model. Suppose that the ith cluster center is $\mathrm{c}_{\mathrm{i}}$ in an $M$ dimensional space. It can be decomposed into two component vectors $\mathrm{p}_{\mathrm{i}}$ and $\mathrm{q}_{\mathrm{i}}$, where $\mathrm{p}_{\mathrm{i}}$ is the input part and it contains the first $\mathrm{N}$ elements of $\mathrm{c}_{\mathrm{i}}, \mathrm{q}_{\mathrm{i}}$ is the output part 
and it contains the last M-N elements of $c_{i}$. Then, for a given input vector $\mathrm{x}$, the degree to which fuzzy rule $i$ is formulated and defined by the equation,

$$
\mu_{i}=\exp \left(-\frac{x-p_{i}^{2}}{\left.\frac{z_{z}}{z}\right)^{2}}\right)
$$

More accuracy can be obtained using gradient descent or other advanced derivative-based optimization schemes for further refinement in rule formation.

Theses algorithms are used in the present work to generate FIS1 and FIS2.

\section{SIMULATION}

GENFIS1 and GENFIS2 are the basic building blocks or modules of ANFIS which uses the algorithms for data partitioning and clustering .It helps in covering the entire input space to classify the samples. Network structure generated using both GENFIS1 and GENFIS2 consists of input, output and rules and the methods used for the rules. Finally defuzzification is done to get the crisp output. 7 input gas concentrations with 5 types of membership functions and one output with linear transfer function are used in this work. Mapping of the input and output from the available data using fuzzy logic is presented. The structure of ANFIS by GENFIS1 module is given below. In case of GENFIS2 all the system input, output, methods used for generating rules and defuzzification remains same except rule structure. In this work the module generated 19 rules as compared to GENFIS1 which has generated 2187 rules, resulting into the complicated structure occupying more memory space. Network structure generated using GENFIS1 consists of the following information and is shown in figure 4.

And Method 'prod'; or Method: 'max'; defuzzification Method: 'wtaver' are used in the work on ANFIS with 3 membership functions for each input.7 gas concentrations are used as inputs and singular output.

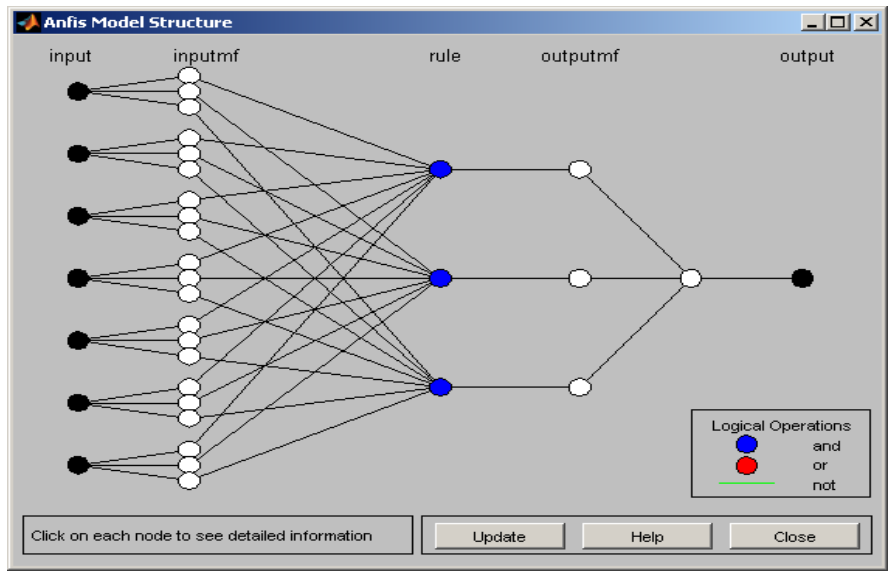

Fig. 4. ANFIS Structure Generated by GENFIS1 


\section{RESULTS AND DISCUSSION}

The well designed TSK-model of ANFIS was trained with set parameters on 563 transformer oil samples using g- bell, triangular, trapezoidal, Gaussian and Gaussian2 membership functions for input and a linear transfer function for the output.

And method is used for input and weight average for the output during defuzzification. The ANFIS was trained and the RMSE as the performance measure for all the input membership functions was obtained separately. The diagnosis performance of both the modules in terms of RMSE and accuracy has been shown in table1.The trained GENFIS1 module with 7 inputs, 1 output and 3 membership functions automatically generated 2187 rules. The training of the modules was carried out to determine the network structure, diagnosis error in terms of RMSE. The diagnosis results of g-bell MF using GENFIS1 are presented and shown in figure 5 and 6 . However other membership functions were also employed and the diagnosis results of all are summarized in table1. GENFIS2 with subtractive clustering algorithm and g-bell membership functions has been employed on the similar number of oil samples as used in GENFIS1. This module generated only 19 rules. This might have deleted the redundant rules up to some extent. Details of its structure and performance are shown in figures 7-9.

The drawback of GENFIS1 is that, it takes more time to converge and is slow, but performs better in diagnosis. In contrast GENFIS2 generated less number of rules to map the input and output data, in which the clustering of data might have helped in reaching the better diagnosis performance. The performance of both the modules is superior; however GENFIS 2 is most fitted to the proposed approach.

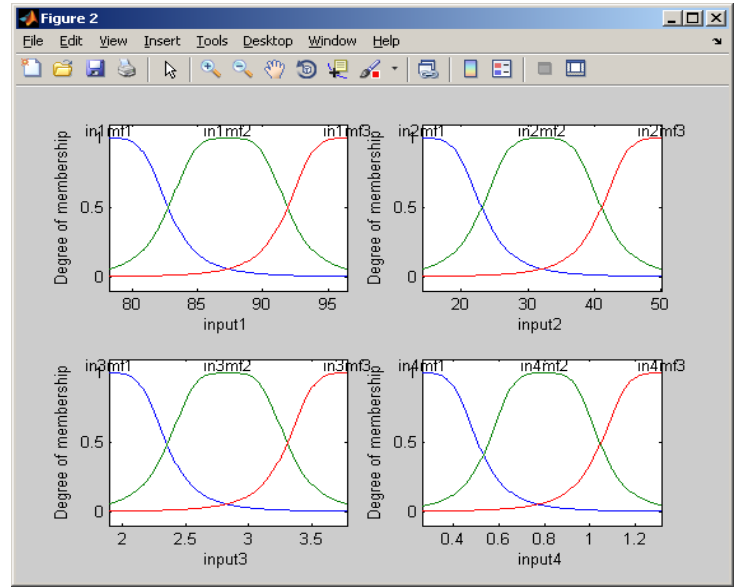

Fig. 5. G-Bell Membership Function

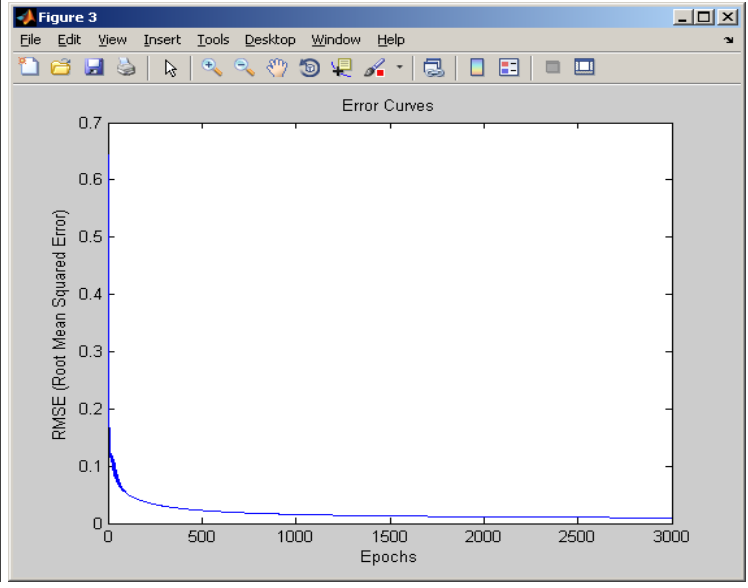

Fig. 6. Error Curve Vs. Epochs (Performance Curve) 
Electrical \& Computer Engineering: An International Journal (ECIJ) Volume 4, Number 1, March 2015

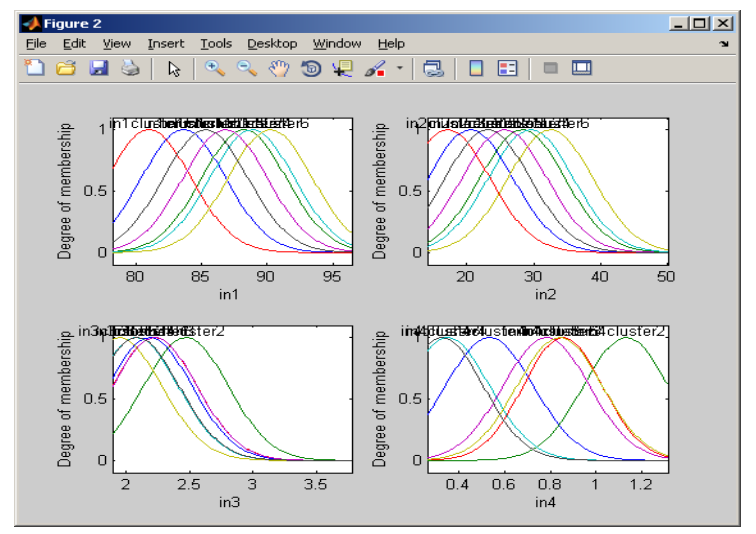

Fig. 7. G-Bell Membership Function in GENFIS2

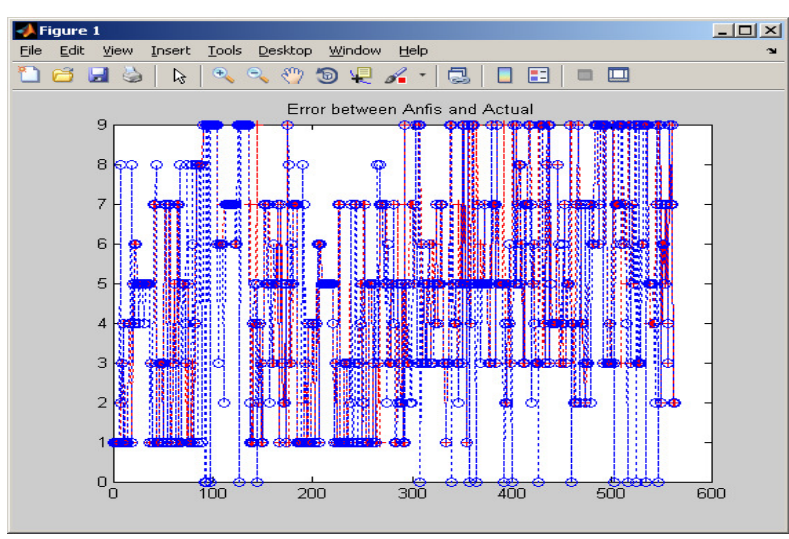

Fig.8.Error between ANFIS and Actual (GENFIS2)

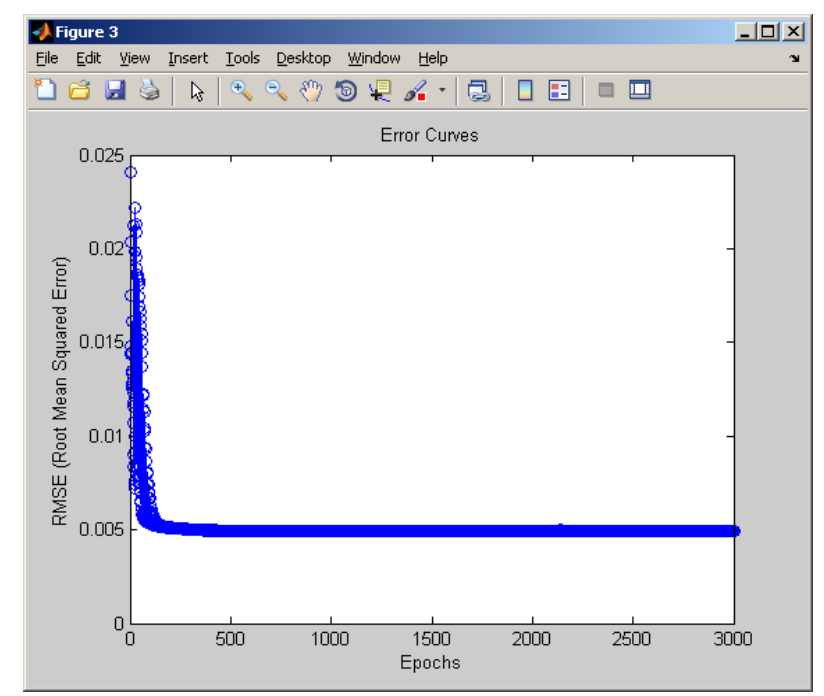

Fig.9. Error Curve Vs. Epochs (Performance Curve)

Table 1.Results of Diagnosis by ANFIS

\begin{tabular}{|c|c|c|c|c|c|c|}
\hline SR.No. & $\begin{array}{c}\text { ANFIS } \\
\text { Model }\end{array}$ & Type of MF & $\begin{array}{c}\text { No. of } \\
\text { iterations }\end{array}$ & RMSE & $\begin{array}{c}\text { Percentage } \\
\text { accuracy }\end{array}$ & No. of rules \\
\hline 1 & GENFIS1 & Trapezoidal & 3000 & 0.003542 & 99.98 & 2187 \\
\hline 2 & GENFIS1 & g-bell & 3000 & 0.0097 & 99.90 & 2187 \\
\hline 3 & GENFIS1 & Gaussian & 3000 & 0.01270 & 99.12 & 2187 \\
\hline 4 & GENFIS1 & Gaussian 2 & 3000 & 0.0100 & 99.10 & 2187 \\
\hline
\end{tabular}


Electrical \& Computer Engineering: An International Journal (ECIJ) Volume 4, Number 1, March 2015

\begin{tabular}{|c|c|c|c|c|c|c|}
\hline 5 & GENFIS1 & Triangular & 3000 & 0.01444 & 99.10 & 2187 \\
\hline 6 & GENFIS2 & g-bell & 3000 & 0.00493 & 99.23 & 19 \\
\hline
\end{tabular}

\section{CONCLUSIONS}

Investigations on the transformer fault diagnosis using ANFIS were carried using both the GENFIS1 and GENFIS2 modules. Both the modules provide comparable performance with respect to the diagnosis error and accuracy. However GENFIS1 is slow in convergence as compared to GENFIS2 and generates more number of rules. This makes the system structure more complex. GENFIS2 has better diagnosis accuracy and less number of rules. It has been identified as the most fitted module of ANFIS for the proposed fault diagnosis of power transformer. Earlier work of the authors was on similar samples but only the method used was grid partitioning with $\mathrm{g}$ bell membership function in the input. It has been enhanced with as many membership functions in the input using grid partitioning and also the subtractive clustering so as to obtain the comparative features of both the modules with better diagnosis performance.

\section{ACKNOWLEDGEMENT}

Authors wholeheartedly thank M/S.B.R.Industrial Services for providing the DGA samples of power transformers. Without sufficient transformer oil samples the work on transformer fault diagnosis would not have been possible.

\section{REFERENCES}

[1] Kelly J.J., "Transformer fault diagnosis by dissolved gas analysis," IEEE Transactions on Industry Applications, Vol.16, No.4, pp.777-782, Dec.1980.

[2] Rogers, R.,IEEE and IEC codes to interpret incipient faults in transformer, using gas in oil analysis. IEEE Transactions on Electr.Insul.,Vol.13,No.5, pp.349-354, October 1978.

[3] R. Naresh, Veena Sharma \& Manisha Vashishth, "An Integrated Neural Fuzzy Approach for Fault Diagnosis of Transformers," IEEE Transactions on Power Delivery,Vol.23,No.4, pp.2017-2024, Oct. 2008.

[4] Vladimiro Miranda, Adriana Rosa Garcez Castro, "Improving The IEC Table For Transformer Failure Diagnosis With Knowledge Extraction From Neural Networks," IEEE Transactions on Power Delivery ,Vol.20,No.4, pp.2509-251, October 2005.

[5] Su,Q.,Mi,C.,lai,L.L., and Austin P., "A Fuzzy Dissolved Gas Analysis Method for the Diagnosis of Multiple Incipient Faults in a Transformer," IEEE Transactions on Power Systems,Vol.15,No.2, , pp.593-598, May 2000.

[6] Rahmatollah Hooshmand and Mahdi Banejad, "Application of Fuzzy Logic in Fault Diagnosis in Transformers Using Dissolved Gas Analysis based on Different Standards," World Academy of Science, Engineering and Technology, Vol.17, pp.157-161, December 2006.

[7]C.S.Chang,C.W.Lim,Q Su, "Fuzzy- Neural Approach for Dissolved Gas Analysis of Transformer Fault Diagnosis," In the proceedings of Australian Universities Power Engineering Conference(AUPEC-2004), Brisbane, Australia, 26-29 September 2004.

[8] lee,J.P.,etal., "Diagnosis of power transformer using fuzzy clustering and radial basis function neural network," In the proceedings of 2006 Joint International Conference on Neural Networks, Vancouver,BC,Canada , July16-21, pp.1398-1404. 
[9] Hamed Malek et al., "Three new fuzzy neural networks learning algorithms based on clustering, training error and genetic algorithm,"Appl.Intell,Springer Science+Business Media,LLC2011,DOI10.1007/s10489-011-0327-7.

[10] Rahmat Allah Hooshmand, Moein Parastegari and Zohreh Forghani, "Adaptive Neuro-Fuzzy Inference System Approach for Simultaneous Diagnosis of the Type and Location of Faults in Power Transformers," IEEE Electrical Insulation Magazine,2012,pp.32-42.

[11] Diego Roberto Morais and Jacqueline Gisèle Rolim , "A Hybrid Tool for Detection of Incipient Faults in Transformers Based on the Dissolved Gas Analysis of Insulating Oil,"IEEE TRANSACTIONS ON POWER DELIVERY, VOL. 21, NO. 2, APRIL 2006,PP.673-680.

[12] S.Rajsekaran, G.A.Vijayalakshmi Pai, Neural networks, fuzzy logic and genetic algorithms-Synthesis and applications. PHI Learning private limited, New Delhi,10th edition ,2008.

[13] J.-S.R.Jang, C.-T.Sun and E.Mizutani, Neuro-fuzzy and soft computing- A computational approach to learning and machine intelligence. Pearson education, Inc., Second impression, 2008.

\section{Authors Biography}

Nandkumar Wagh obtained his B.E.(Electronics \& Power) and M.E.(EPS) degree from Govt.College of Engineering, Amravati(M.S.) in the year 1986 and 1996 respectively. Now he is pursuing his Doctoral degree from Maulana Azad National Institute of Technology, Bhopal (M.P.).Presently he is working as Associate Professor in Electrical Engineering department at DES's College of Engineering and Technology, Dhamangaon Rly (M.S.) India. He occupied various positions to serve the engineering institutes for about 27 years. Power system operation and control, applications of AI in

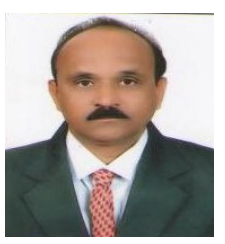
electrical power system and electrical drives are his prime areas of research interest. He has published many papers in national and international journals and conferences.

D.M. Deshpande obtained his Ph D from IIT, Roorkee (India). He is currently working with SIRTS, Bhopal as Professor of Electrical Engineering. Prior to this appointment he was associated with Maulana Azad National Institute of Technology; Bhopal (India), where he served as Professor in Electrical Engineering department. He is having a vast experience in teaching and industry. He has published many research papers in national and international journals and conferences. 